OPEN ACCESS

Edited by:

Sung-Rae Cho,

Yonsei University,

South Korea

Reviewed by:

Joseph Bleiberg,

National Intrepid Center of

Excellence (NICOE),

United States

Francisco Capani,

University of Buenos

Aires, Argentina

*Correspondence:

Mauro Mancuso

m.mancuso62@gmail.com

Specialty section:

This article was submitted

to Neurotrauma,

a section of the journal

Frontiers in Neurology

Received: 28 September 2017 Accepted: 12 February 2018

Published: 28 February 2018

Citation:

Mancuso M, Demeyere N, Abbruzzese L, Damora A, Varalta V, Pirrotta F, Antonucci G, Matano A, Caputo M, Caruso MG, Pontiggia GT, Coccia M, Ciancarelli I, Zoccolotti P and The Italian OCS Group (2018) Using the Oxford Cognitive Screen to

Detect Cognitive Impairment in Stroke Patients: A Comparison with the Mini-Mental State Examination.

Front. Neurol. 9:101.

doi: 10.3389/fneur.2018.00101

\section{Using the Oxford Cognitive Screen to Detect Cognitive Impairment in Stroke Patients: A Comparison with the Mini-Mental State Examination}

\author{
Mauro Mancuso ${ }^{1 *}$, Nele Demeyere ${ }^{2}$, Laura Abbruzzese', Alessio Damora', \\ Valentina Varalta ${ }^{3}$, Fabio Pirrotta ${ }^{1}$, Gabriella Antonucci ${ }^{4,5}$, Alessandro Matano, \\ Marina Caputo ${ }^{6}$, Maria Giovanna Caruso ${ }^{7}$, Giovanna Teresa Pontiggia ${ }^{8}$, Michela Coccia 9 , \\ Irene Ciancarelli ${ }^{10}$, Pierluigi Zoccolotti ${ }^{4,5}$ and The Italian OCS Group \\ ${ }^{1}$ Tuscany Rehabilitation Clinic, Montevarchi, Italy, ${ }^{2}$ Cognitive Neuropsychology Centre, Department of Experimental \\ Psychology, University of Oxford, Oxford, United Kingdom, ${ }^{3}$ Neuromotor and Cognitive Rehabilitation Research Center, \\ Department of Neurological, Biomedical and Movement Sciences, University of Verona, Verona, Italy, ${ }^{4}$ Department of \\ Psychology, Sapienza University of Rome, Rome, Italy, ${ }^{5}$ Neuropsychology Center, IRCCS Santa Lucia Foundation, Rome, \\ Italy, ${ }^{6}$ Neurological Rehabilitation Unit, Auxilium Vitae Volterra, Volterra, Italy, ${ }^{7}$ Physical and Rehabilitation Medicine, \\ Department of Medical and Surgical Sciences "Magna Graecia" University, Catanzaro, Italy, ${ }^{8}$ Neuropsychology Unit, National \\ Health Service, Bari, Italy, ${ }^{9}$ Department of Neuroscience, Neurorehabilitation Clinic, Azienda Ospedaliero-Universitaria \\ Ospedali Riuniti, Ancona, Italy, ${ }^{10}$ Department of Life, Health and Environmental Sciences, University of L'Aquila, Nova Salus \\ s.r.l., L'Aquila, Italy
}

Background: The Oxford Cognitive Screen (OCS) was recently developed with the aim of describing the cognitive deficits after stroke. The scale consists of 10 tasks encompassing five cognitive domains: attention and executive function, language, memory, number processing, and praxis. OCS was devised to be inclusive and un-confounded by aphasia and neglect. As such, it may have a greater potential to be informative on stroke cognitive deficits of widely used instruments, such as the Mini-Mental State Examination (MMSE) or the Montreal Cognitive Assessment, which were originally devised for demented patients.

Objective: The present study compared the OCS with the MMSE with regards to their ability to detect cognitive impairments post-stroke. We further aimed to examine performance on the OCS as a function of subtypes of cerebral infarction and clinical severity.

Methods: 325 first stroke patients were consecutively enrolled in the study over a 9-month period. The OCS and MMSE, as well as the Bamford classification and NIHSS, were given according to standard procedures.

Results: About a third of patients (35.3\%) had a performance lower than the cutoff $(<22)$ on the MMSE, whereas $91.6 \%$ were impaired in at least one OCS domain, indicating higher incidences of impairment for the OCS. More than $80 \%$ of patients showed an impairment in two or more cognitive domains of the OCS. Using the MMSE as a standard of clinical practice, the comparative sensitivity of OCS was 100\%. Out of the 208 patients with normal MMSE performance 180 showed impaired performance in at least one domain of the OCS. The discrepancy between OCS and MMSE was particularly strong for patients with milder strokes. As for subtypes of cerebral infarction, fewer 
patients demonstrated widespread impairments in the OCS in the Posterior Circulation Infarcts category than in the other categories.

Conclusion: Overall, the results showed a much higher incidence of cognitive impairment with the OCS than with the MMSE and demonstrated no false negatives for OCS vs MMSE. It is concluded that OCS is a sensitive screen tool for cognitive deficits after stroke. In particular, the OCS detects high incidences of stroke-specific cognitive impairments, not detected by the MMSE, demonstrating the importance of cognitive profiling.

Keywords: stroke, cognitive assessment, Oxford Cognitive Screen, Mini-Mental State Examination, cognitive screening

\section{INTRODUCTION}

Stroke is the second most common cause of death and the third most common source of disability $(1,2)$. Its prevalence and incidence increases with age representing the leading cause of disability in the elderly (2). Patients with stroke have cognitive deficits in a very high proportion of cases [e.g., Ref. (1)], although variables estimates are reported. The differences are likely due to variability in sample characteristics, assessment methods, definitions of impairment, and time interval since stroke onset (1). As cognitive assessment is time consuming, physicians often use smart tools to assess cognitive impairment in stroke survivors that need little time but are often of limited use to highlight cognitive dysfunction, typically yielding relatively low-prevalence rates, below $25 \%(3,4)$. On the opposite, more detailed neuropsychological assessments of domain-specific cognitive impairments consume more time but are better at detecting cognitive impairment, highlighting higher occurrences, ranging from 35 to $92 \%(5-8)$. Language, spatial attention, memory, praxis, executive function, and speed of processing are the main impaired cognitive domains (5). Moreover, psychiatric comorbidities such as depression and delirium often occur after a stroke (9). Cognitive deficits interfere with rehabilitation and represent a negative prognostic factor $(10,11)$, impacting on activities of daily living, quality of life, and return to work (12).

Stroke guidelines recommend the importance of early cognitive diagnosis in order to plan tailored rehabilitation programs (13). Tools, such as the Mini-Mental State Examination [MMSE; (14)] and the Montreal Cognitive Assessment [MoCA; (15)], are widely used as a practical solution to briefly assess cognition poststroke. However, these instruments were devised for evaluation of patients with dementia and only provide a "domain-general" cognitive score with a single cutoff for impairment. The present study describes the use of a newly devised instrument, the Oxford Cognitive Screen (OCS), against one of these two reference tools, namely the MMSE; in a parallel study, we examined the effectiveness of the OCS against the MoCA (16).

Interest in using the MMSE as a comparison chiefly stems from its wide use; indeed, it is one of the most widely tests used in clinical practice. Early reviews emphasized the reliability and construct validity of the MMSE to capture moderate-to-severe cognitive impairment (17). However, the limits of the MMSE are also well-known particularly in the assessment of stroke patients $(18,19)$. In spite of this, the MMSE is still one of the instruments which is most widely used nowadays in clinical settings to detect global cognitive impairment in patients with stroke (20-30). In particular, it is used as a diagnostic index in the stroke units to plan the rehabilitation interventions as well as in the identification of cognitive profiles after non-dementia cerebro-vascular events $(21,29,31-33)$.

The key problem in using the MMSE to assess stroke sequelae is that it does not explicitly assess common post-stroke domainspecific impairments such as neglect, executive function, apraxia, and aphasia. Indeed, performance on the MMSE can be confounded by co-occurring difficulties in these domains. For example, a patient with expressive aphasia will maximally score 4 points (out of a maximum 30) as the large majority of tasks require spoken language. This would lead to a potential misclassification of patients as "dementia" where there is a specific language impairment. Similarly, specific cognitive impairments may be "missed" in patients with stroke. This lack of specificity contrasts the indications of several clinical guidelines which emphasize the need to assess performance across different domains of cognition after stroke [e.g., Ref. $(2,34)]$.

The OCS was recently developed with the specific aim of describing the cognitive deficits after stroke (35); OCS was devised to be inclusive and un-confounded by aphasia and neglect. It can be administered within $15 \mathrm{~min}$, can be delivered at the bedside, is easy to administer and score, can be used in relatively acute phase (after 3 days from onset) and provides a "snapshot" of a patient's cognitive profile useful to define the rehabilitative program. The possibility to have separate cutoff for each of the tasks used allows obtaining a cognitive pinpointing strengths and weaknesses of individual stroke patients.

The scale consists of 10 tasks encompassing five cognitive domains: attention and executive function, language, memory, number processing, and praxis. Furthermore, it includes a brief evaluation of visual field defects. Administration is simple and brief (ca. $15 \mathrm{~min}$ ) making it also suitable for immobilized patients. Demeyere et al. (16) provided initial data on a sample of stroke patients indicating the ability of the scale to detect differentiated profiles across the various domains and also reported a greater sensitivity of OCS over MoCA.

In order to assess whether this new instrument provides a sensitive and practical first line assessment supporting wider adoption, the present study aimed to compare the OCS with the MMSE in detecting cognitive symptoms after stroke, thereby providing further data on the sensitivity and specificity of the OCS in the identification of cognitive deficits in a relatively large sample of first stroke patients. We also examined OCS 
performance as a function of subtypes of cerebral infarction [based on the Bamford classification; (36)] and clinical severity [based on the National Institutes of Health Stroke Scale, NIHSS; (37)].

\section{MATERIALS AND METHODS}

\section{Patients}

Fourteen different Italian rehabilitative centers participated in this study. In each center, the patients were consecutively recruited from February to October 2016 and selected based on the following inclusion and exclusion criteria.

- Inclusion criteria: Patients at first episode; both gender; aged from 18 to 90 years; distance from stroke onset within 180 days; patients who were able to give informed consent; ability to concentrate for a minimum of $20 \mathrm{~min}$ (as judged by the care team).

- Exclusion criteria: Patients with a previous stroke; the presence of premorbid psychiatric or neurological disease; distance from onset $<72 \mathrm{~h}$; patients unable to give informed consent; patients without ability to concentrate for $<20 \mathrm{~min}$ (as judged by the care team).

The sample dimension was calculated through a power analysis carried out with the $\mathrm{G}^{\star}$ Power 3.1 program (finale sample tested $n=325$ patients; see below for a description).

The research was approved by the Tuscany-South East Vast Area Ethical Committee (n. 376/2015); all patients signed the informed consent.

\section{Cognitive Screening Tests}

The OCS (35) assesses five cognitive domains: attention and executive function, language, memory, number processing, and praxis. Furthermore, visual field deficits are also assessed.

A brief description of tests according to each domain follows (in brackets the order of sub-test presentation):

\section{- Language domain:}

- Picture naming (1): The patient is requested to name four pictures in order to assess the expressive language.

- Semantics (2): The patient has to point the picture asked, choosing between four pictures simultaneously shown.

- Sentence reading (5): One centrally aligned 15-word long sentence is presented and the participant is asked to read it aloud. The sentence is later used for an unanticipated verbal memory task.

- Memory domain:

- Orientation (3): The participant is asked about which city is in, the time of the day, the month, and the year.

- Recall and recognition (10): The patient is asked to recall the sentence previously read in the "sentence reading task." If the patient is unable to recall the sentence, a page with four options for each irregular word is shown. A further four multiple-choice questions are then given to address nonverbal, episodic memory through task recall.
- Number processing domain:

- Number writing (6): The patient is asked to write down the numbers heard.

- Calculation task (7): The patient is required to make two additions and two subtractions.

- Attention and executive function domain:

- Broken hearts (8): A page with 150 semi-randomly positioned hearts ( 50 full hearts and 50 broken on the left and 50 on the right) is presented. The task is to cancel out all complete hearts while not crossing out hearts broken on the left or on the right with a 3-min time limit.

- Trails task (11): Stimuli are pages with circles and squares of different sizes. The two baseline tasks require drawing a line between either circles or squares going down in size and alternating between circles and squares, again going down in size (largest square to largest circle to next largest square, etc.).

- Praxis domain:

- Imitating meaningless gestures (9): This test requires to patient to mirror imitate, with their better hand, a series of meaningless hand and finger actions made by the examiner.

- Visual field (4): The examiner faces the participant and raises both hands, first to the participant's upper visual fields, and then to the lower fields asking to wiggle the fingers of the left or right hand.

The OCS can be administered in about 15 min and can be delivered at the bedside, whenever necessary. The Italian version of the scale, with normative data on a sample of 20 - to 80 -year-old individuals, was used (38).

The MMSE is a widely used tool for cognitive assessment. As a domain-general cognitive screen, the MMSE ultimately returns a pass/fail judgment, based on a single overall score. The instrument can be divided into two main sections: the first requires only verbal feedback and includes the evaluation of orientation, memory, and attention. The second part assesses the naming skills, to follow verbal and written commands, write spontaneously a sentence, and copy a complex polygon. Although the MMSE contains subsections, there are no sub-domain cutoffs. The total maximum score is 30 and the total time of administration is about $10 \mathrm{~min}$. Normative values, adjusted for age and education, are available for the Italian elderly population and the cutoff $<22$ was established for pathological performance (14).

The OCS and MMSE were presented on the same day; order of presentation of the two tests was counterbalanced on an $\mathrm{ABAB}$ basis for each rehabilitation center. Tests were administered in a quiet and comfortable setting.

\section{Clinical Scales}

The NIHSS was designed to evaluate stroke severity. It consists of 11 items, the total score ranging from a minimum of 0 (normal neurological functioning) to a maximum of 42 (severe neurological damage). The following categories are considered: minor stroke $=1-4$, moderate $=5-15$, moderate to severe $=16-20$, severe $=21-42$. The time of administration is about $5 / 8 \mathrm{~min}(37)$. 
The Bamford Classification (36) allows clustering patients with cerebral infarction according to some distinctive features, placing them into four groups: Lacunar Infarcts, Total Anterior Circulation Infarcts, Partial Anterior Circulation Infarcts, and Posterior Circulation Infarcts (POCI) on the basis of the signs and symptoms.

\section{Statistics}

For descriptive purposes, we examined the percentage (\%) of patients to whom the OCS could not be administered, as well as the $\%$ of patients falling in one or more domains of the scale.

In order to examine the significance of the association between different kinds of classification, we used Fisher's exact test for categorical data when expected values in at least one cell was below 5 . This was the case for the Bamford classification, OCS, and MMSE. Conversely, $\chi^{2}$ tests were used to examine contingency tables with expected values always above 5 , as in the case of NIHSS values.

\section{RESULTS}

Over the 9 months of data collection, we recruited a total of 325 patients (178 males and 147 females). The mean age was 69.46 years $(\mathrm{SD}=12.53)$ while years of education averaged 9.07 years $(\mathrm{SD}=4.52)$. The average time from stroke was 33.9 days $(\mathrm{SD}=41.8)$. Demographic and clinical features of the sample are given in Table 1. Based on CT scan data 278 patients had ischemic and 44 hemorrhagic stroke etiologies. Unilateral lesions were found in 306 patients: 122 had left-hemisphere damage (LHD) and 184 right-hemisphere damage (RHD). Only 19 patients had bilateral (midline) or cerebellar lesions.

The OCS proved generally easy to administer and only for three patients $(0.9 \%)$ the scale could not be given at all. In a few cases, some tests could not be administered (see Table 2); the main reasons for this were severe visual or motor impairment, fatigue or inability to understand the instructions due to severe global aphasia. The MMSE could not be administered to six patients.

TABLE 1 | Characteristics of the sample.

\begin{tabular}{llcc}
\hline & Category & $\begin{array}{c}\text { No. of patients } \\
\text { (325) }\end{array}$ & \% \\
& Male & 178 & 54.7 \\
Gender & Female & 147 & 45.2 \\
\hline Etiology & Ischemic & 278 & 85.5 \\
& Hemorrhagic & 44 & 13.5 \\
\hline Lesion lateralization & Unilateral left hemisphere & 122 & 37.5 \\
& Unilateral right hemisphere & 184 & 56.6 \\
& Bilateral/cerebellar & 19 & 5.8 \\
\hline Vascular territory affected & TACl & 58 & 17.8 \\
for ischemic patients: & LACl & 76 & 23.3 \\
Bamford classification & PACl & 91 & 28 \\
$(n=274)$ & POCl & 54 & 16.6 \\
\hline Stroke severity: & Minor & 171 & 52.6 \\
NIHSS & Moderate & 136 & 41.8 \\
& Moderate to severe & 13 & 4 \\
& Severe & 4 & 1.2
\end{tabular}

About a third of patients $(n=115 ; 35.3 \%)$ had a performance lower than the cutoff $(<22)(14)$ on the MMSE, whereas $91.6 \%$ $(n=295)$ were impaired in at least one OCS domain (Table 3), indicating higher incidences of impairment for the OCS than MMSE (Fisher's exact $p<0.0001$ ). More than $80 \%$ of patients $(n=263 ; 81.6 \%)$ showed an impairment in two or more cognitive domains of the OCS. Using the MMSE as a standard of clinical practice, the comparative sensitivity of OCS was $100 \%$, indicating that no patients had an impairment in MMSE but not in OCS. Infact, 180 patients with cognitive deficit were detected as positive to OCS but not to MMSE, indicating that OCS did not show false negative.

Out of the 208 patients with normal MMSE performance (i.e., a score of at least 22), 180 showed impaired performance in at least one domain of the OCS (Table 3 ). Note the high proportion

TABLE 2 | Number and percentage of patients for whom Oxford Cognitive Screen tests could not be administered.

\begin{tabular}{llcc}
\hline Domain & Tasks & $\boldsymbol{n}$ & $\%$ \\
\hline Language & Picture naming & 4 & 1.2 \\
& Semantics & 3 & 0.9 \\
& Sentence reading & 5 & 1.2 \\
\hline Memory & Orientation & 3 & 0.9 \\
& Recall and recognition & 3 & 0.9 \\
\hline Number & Episodic memory & 3 & 0.9 \\
\hline Perception & Number writing & 5 & 1.5 \\
\hline Spatial attention & Calculation & 3 & 0.9 \\
\hline Praxis & Visual field & 5 & 1.5 \\
\hline Executive function & Hearts cancelation & 17 & 5.2 \\
& Space asymmetry & 21 & 6.4 \\
& Object asymmetry & 21 & 6.4 \\
\hline & Imitation & 5 & 1.5 \\
\hline
\end{tabular}

TABLE 3 | Number (and percentage) of patients who passed the cutoff for Mini-Mental State Examination $(n=208)$ but failed in one or more of the Oxford Cognitive Screen tests.

\begin{tabular}{lllr}
\hline Domain & Task & $\boldsymbol{n}$ & $\%$ \\
\hline Language & Picture naming & 71 & 34.1 \\
& Semantics & 10 & 4.8 \\
& Sentence reading & 71 & 34.1 \\
\hline Memory & Orientation & 17 & 8.1 \\
& Recall and recognition & 61 & 29.3 \\
& Episodic memory & 65 & 31.2 \\
\hline Number & Number writing & 35 & 16.8 \\
\hline Perception & Calculation & 76 & 36.5 \\
\hline Spatial attention & Visual field & 23 & 11 \\
\hline Praxis & Hearts cancelation & 95 & 45.6 \\
Executive function & Space asymmetry & 43 & 20.6 \\
& Object asymmetry & 14 & 6.7 \\
\hline & Imitation & 33 & 15.8 \\
& Baseline score & 77 & 37 \\
& Shifting score & 65 & 31.2
\end{tabular}


of patients (about one-third or more) with impaired performance in picture naming and sentence reading (language domain), calculation (number processing domain), recall and recognition and episodic memory tests (memory), and hearts cancelation (attention domain). Only 27 patients were not impaired in both cognitive scales.

Most patients ( $n=266 ; 82.1 \%$ ) had a motor, sensory, or cognitive deficit on the NIHSS (proportion of patients as a function of stroke severity is presented in Table 1). There was a weak, but significant, inverse correlation between the severity of the NIHSS and MMSE corrected score $(r=-0.186, p=0.001)$. Incidence of impairment on the OCS was significantly higher than that in the MMSE in the minor $\left(\chi_{(1)}^{2}=41.18, p<0.0001\right)$ and moderate $\left(\chi_{(1)}^{2}=32.82, p<0.0001\right)$ categories but not in the moderatesevere category $\left(\chi_{(1)}^{2}=1.16, p=0.28\right.$; see Figure 1$)$.

In relationship to the Bamford Classification, the frequency of impairments in OCS domains was higher than the MMSE impairments for all categories (all Fisher's exact tests at least $p<0.01)$. When considering the number of domain impairments on OCS, fewer patients demonstrated widespread impairments (i.e., more than three domains) in the POCI category than in the other categories (see Figure 2).

With regards to lesion side, there were $37.5 \%(n=122)$ patients with LHD and $56.6 \%(n=184)$ patients with RHD. The remaining patients had bilateral and/or cerebellar damage. Among LHD patients, $43.4 \%$ were deficient in the MMSE, while $91.8 \%$ were impaired in at least one OCS domain. Among RHD patients, 32\% were deficient in the MMSE, while $90.2 \%$ were impaired in at least one OCS domain.
Incidence of impairments in the MMSE and in the different OCS domains/tests is reported in Table 4 separately for the overall sample, the LHD and RHD patients. LHD patients had higher frequency of impairments in the language domain (sentence reading test) and in the memory domain (recall and recognition test). In the broken hearts test, a higher percentage of RHD patients showed inattention for left space while a higher percentage of LHD patients showed a higher percentage of right inattention for the right space. Finally, there was a trend for RHD patients to be more impaired in the shifting score (executive function domain). As for the MMSE, RHD patients were more frequently impaired than LHD patients.

\section{DISCUSSION}

Our study examined the detection of cognitive symptoms after a first stroke by comparing two scales, the widely used MMSE, and a new instrument, the OCS. The results showed a much higher incidence of cognitive impairment with the OCS (91\% at least one domain impairment) than with the MMSE (35\%), and demonstrated no false negatives for OCS vs MMSE (giving OCS a comparative sensitivity of $100 \%)$. These data support recent reports on the under-detection of post-stroke cognitive impairments of MMSE $(18,39)$ and further highlight the shortcomings of using tests designed for dementia $(16,40)$ within a stroke population. In particular, in a parallel study we demonstrated the greater sensitivity of the OCS scale over the MoCA scale, another instrument originally devised for the detection of dementia symptoms. In this respect, it may be noted that

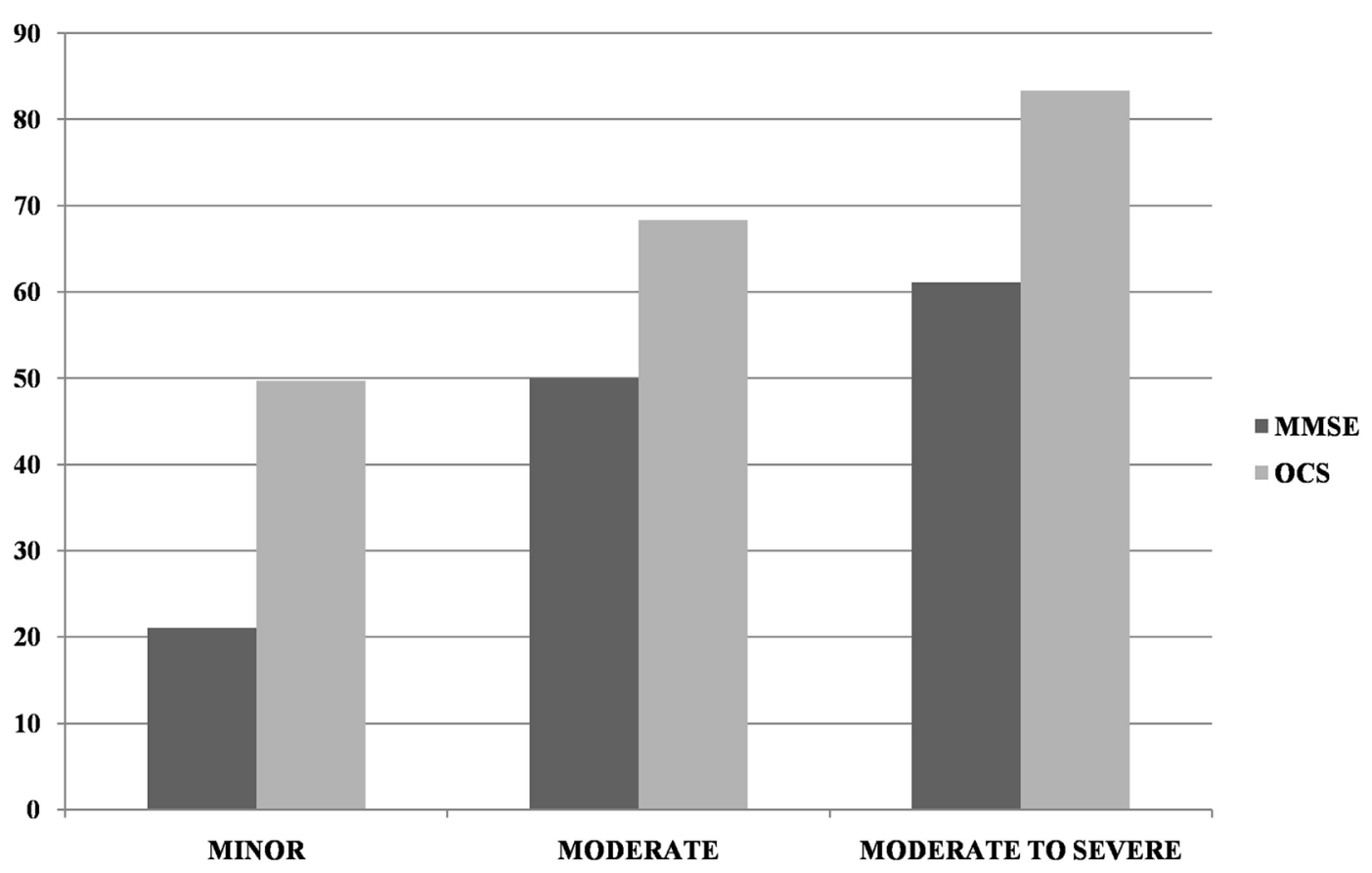

FIGURE 1 | Incidence of impairment at Mini-Mental State Examination (MMSE) and Oxford Cognitive Screen (OCS) (at least one domain) as a function of NIHSS severity (minor = NIHSS 1-4, moderate $=5-15$, moderate-severe $=16-20$ ). Too few patients were in the severe NIHSS category to allow for reliable comparisons. 


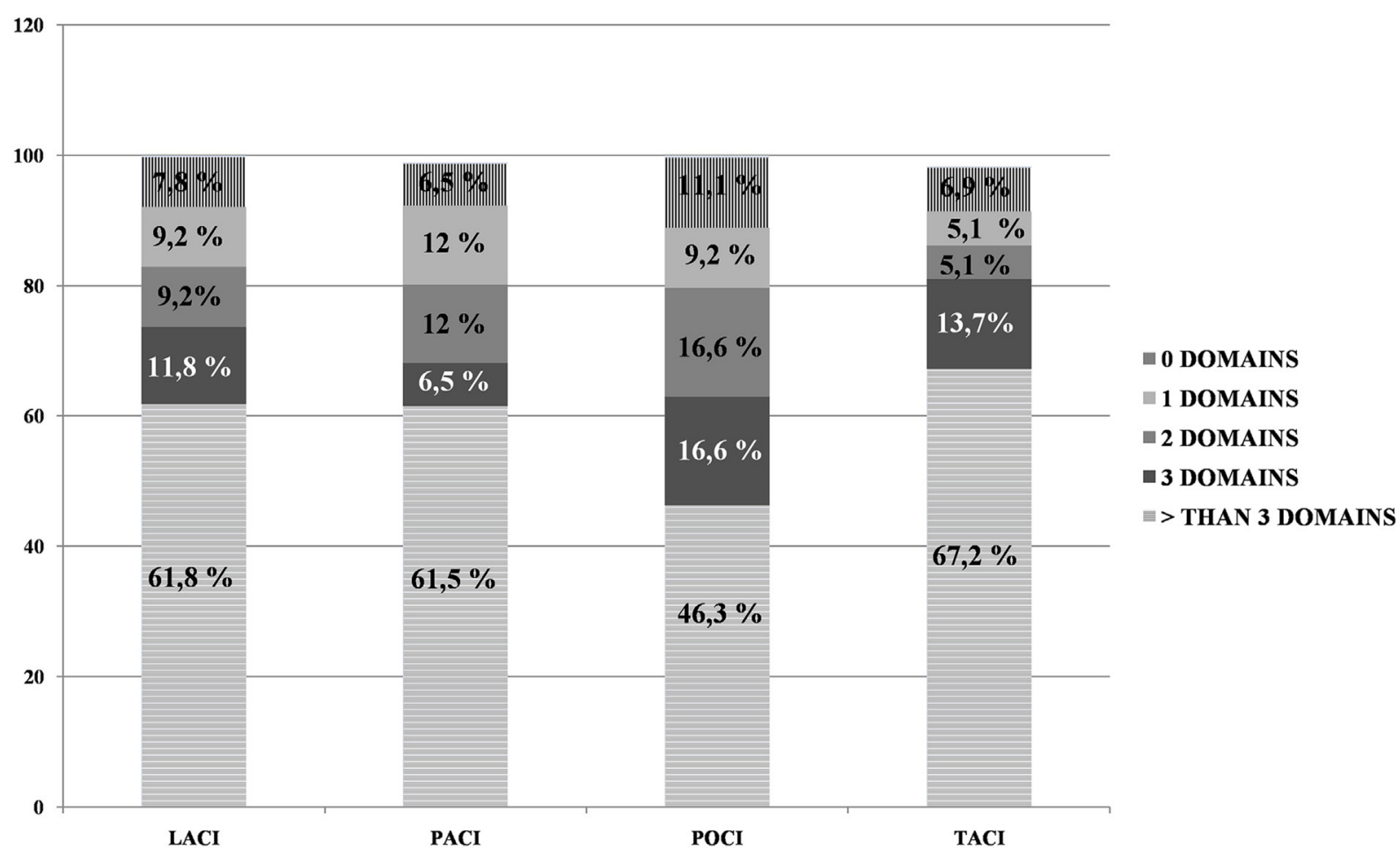

FIGURE 2 | Percentage of Oxford Cognitive Screen domain impairments for each of the four Bamford categories

TABLE 4 | Percentage of patients obtaining a pathological score in the Mini-Mental State Examination (MMSE) and Oxford Cognitive Screen (OCS) tests as a function of lesion lateralization.

\begin{tabular}{|c|c|c|c|c|c|c|}
\hline \multirow[t]{2}{*}{ Screen } & \multirow[t]{2}{*}{ Cognitive domain } & \multirow[t]{2}{*}{ Task } & \multicolumn{4}{|c|}{ Lateralization } \\
\hline & & & Overall (\%), $n=325$ & Unilateral left (\%), $n=122$ & Unilateral right (\%), $n=184$ & $p$-Value \\
\hline MMSE & Overall score & Cutoff $=22$ & 35.3 & 43.4 & 32 & 0.039 \\
\hline \multirow[t]{19}{*}{ OCS } & Language & Picture naming & 43.6 & 47.5 & 40.7 & 0.156 \\
\hline & & Semantics & 11.08 & 10.6 & 12.5 & 0.72 \\
\hline & & Sentence reading & 49.8 & 57.3 & 45.1 & 0.019 \\
\hline & Memory & Orientation & 21.8 & 22.1 & 22.8 & 1.000 \\
\hline & & Recall and recognition & 47.0 & 56.5 & 42.9 & 0.013 \\
\hline & & Episodic memory & 48.3 & 48.3 & 47.2 & 0.725 \\
\hline & Number & Number writing & 36 & 40.1 & 34.2 & 0.223 \\
\hline & & Calculation & 50.7 & 55.7 & 48.3 & 0.158 \\
\hline & Perception & Visual field & 15.6 & 11.4 & 18.4 & 0.147 \\
\hline & Spatial attention & Hearts cancelation & 55.6 & 50 & 60.3 & 0.121 \\
\hline & & Space asymmetry & 31.3 & 28.6 & 35.3 & 0.316 \\
\hline & & Left inattention $>3$ & 20 & 20.4 & 26.09 & 0.000 \\
\hline & & Right inattention $<-3$ & 14 & 12.3 & 10.3 & 0.018 \\
\hline & & Object asymmetry & 6.7 & 7.3 & 4.8 & 0.456 \\
\hline & & Left inattention > 2 & 10.1 & 7.3 & 12.5 & 0.122 \\
\hline & & Right inattention $<-2$ & 6.7 & 6.5 & 4.8 & 0.332 \\
\hline & Praxis & Imitation & 28.3 & 29.5 & 27.7 & 0.697 \\
\hline & Executive function & Baseline score & 34.7 & 36.07 & 34.2 & 0.71 \\
\hline & & Shifting score & 32.3 & 25.4 & 36.9 & 0.076 \\
\hline
\end{tabular}

there is some indication of a greater sensitivity of MoCA over the $\operatorname{MMSE}(20,39,41)$; however, there is also counter-evidence pointing to a substantial equivalence between these two instruments [(42); for a review see Ref. (43)]. This is particularly the case when cutoffs adjusted for age and schooling (21) are used
(22) as in the present study. Overall, it appears that cognitive deficits after stroke are detected more effectively by a tool (OCS) selectively devised to capture deficits after stroke than by tools originally devised for dementia, such as MMSE and MoCA. 
The detection of discrepancy between OCS and MMSE was particularly strong for patients with milder stroke, i.e., in the lower NIHSS range. For patients in the moderate-to-severe category, there was no longer a difference between the cognitive screens, as almost all patients had severe cognitive impairments, likely to be detected by any type of cognitive screening. This finding is in keeping with previous observations that MMSE is not sensitive to mild cognitive deficits [e.g., (44)].

Over $80 \%$ of patients showed an impairment in more than one cognitive domain on the OCS, demonstrating the importance of cognitive profiling (45). A recent study highlighted that the MMSE was only able to detect much lower frequencies of cognitive dysfunction [24-50\% and 6-31\%, respectively; (1)]. The OCS detected high incidences of stroke-specific cognitive impairments, not detected by the MMSE: e.g., neglect was present in $31 \%$ of the patients unimpaired on the MMSE. Similarly, $49 \%$ of patients failed in the sentence reading task (Table 4). These stroke-specific problems are not detected in the MMSE as they are considered to be unimpaired within a dementia profile; so, they are not assessed in this screen. Given that such strokespecific deficits may be targeted by specific interventions (e.g., occupational therapy for apraxia, scanning or prism therapies for neglect, specific communication therapies for different language impairments, etc.), domain-level cognitive profiling is likely to be effective for planning individually tailored interventions.

Considering the Bamford classification, fewer patients presented widespread cognitive impairments (i.e., more than three OCS domains) in the POCI category. It is known that POCI stroke infarcts do not affect subcortical structures involving the larger attention networks. This is in line with other studies that indicate that patients with lesions in the vertebro-basilar territory have clinical features that make them different from patients with lesions in the cortical or subcortical areas (46). Infact, the impairment of these posterior areas most frequently cause motor deficits, articulatory speech difficulties, dysphagia, vertigo, nausea or vomiting, facial palsies, or lower cranial nerve deficits (46). In contrast, strokes in these posterior areas result in less disruption of brain regions involved in cognition (47). Thus, patients with ischemia in these areas typically show better cognitive outcomes and cognitive deficits, such as aphasia and neglect, are infrequent (47).

Finally, some features of the OCS scale that indicate its feasibility for clinical use on post-stroke patients should be emphasized. The scale allows detailed screening of the cognitive domains that are compromised following stroke, through the separate assessment of memory, language, number cognition, praxis, executive functions, and attention; visualization of patient's strengths and weaknesses is made possible through reference to separate cutoffs for single subtests. Administration is compatible with the presence of severe language impairments as OCS includes items that do not require language-based answers (e.g., the patient has to indicate the answer among different visual alternatives); similarly,

\section{REFERENCES}

1. Jokinen H, Melkas S, Ylikoski R, Pohjasvaara T, Kaste M, Erkinjuntti T, et al. Post-stroke cognitive impairment is common even after successful clinical recovery. Eur J Neurol (2015) 22:1288-94. doi:10.1111/ene.12743 the influence of left unilateral spatial neglect is minimized by arranging targets vertically whenever appropriate. Finally, the administration is generally short (usually within $15 \mathrm{~min}$ ) and well suited to daily clinical practice, including the patient's bedside. The experience with patients in the present study confirmed these general comments. Only for three patients (i.e., less than 1\%) we could not administer the OCS; in a limited number of cases, individual subtests could not be given (this ranged from 0.9 to $8.6 \%$ depending on the sub-test).

\section{Conclusion}

Results indicate that OCS presents a sensitive screening procedure for cognitive deficits after stroke. In particular, the OCS detects high incidences of stroke-specific cognitive impairments, not detected by the MMSE, demonstrating the importance of cognitive profiling. In view of the wide use of MMSE, we propose that this conclusion calls for a substantial revision of the clinical standards for the screening procedures of cognitive deficits after stroke.

\section{ITALIAN OCS GROUP}

Chiara Beni, Fabio Giovannelli (Arezzo); Ivana Bureca (Rome); Mauro Zampolini, Adonella Benedetti (Perugia); Nicola Smania, Cristina Fonte, Elisa Ghirardi (Verona); Maurizio Iocco (Catanzaro); Federica Galli (Ancona); Laura Prospero, Adriana Gadaleta, Maristella Scattaglia, Franco Valluzzi (Bari); Nicoletta Caputi (L'Aquila); Serena De Pellegrin (Padova); Michelangelo Bartolo (Bergamo), Chiara Zucchella (Verona); Pietro Spinelli, Irene Aprile (Roma); Caterina Pistarini, Valeria Pingue (Pavia); Mirco Soda (Bolzano).

\section{ETHICS STATEMENT}

Comitato Etico Regione Toscana. Area Vasta Sud Est. Prot. 376/ CEAVSE del 17-12-2015.

\section{AUTHOR CONTRIBUTIONS}

MM, ND, PZ, GA, AM, MC, MGC, GP, MC, and IC designed the experiment; all members of the Italian OCS group provided data, LA, FP, AD, and VV collected and analyzed the data; MM, PZ, LA, and FP wrote the paper; MM, ND, PZ, GA, and AM provided critical feedback and gave final approval of the version to be published.

\section{ACKNOWLEDGMENTS}

The OCS scale is freely available for publicly funded clinical and research use. OCS is being licensed via Oxford University Innovations, in order to track uptake. ND was supported by the Stroke Association (reference TSA LECT 2015/02).

2. SPREAD. Stroke Prevention and Educational Awareness Diffusion. 8th ed (2017). AN: L.T.COM.09.2016.1690 cod. Art. 86145856.

3. Liman TG, Heuschmann PU, Endres M, Floel A, Schwab S, KolominskyRabas PL. Changes in cognitive function over 3 years after first-ever stroke and predictors of cognitive impairment and long-term cognitive stability: 
the Erlangen Stroke Project. Dement Geriatr Cogn Disord (2011) 31:291-9. doi: $10.1159 / 000327358$

4. Douiri A, Rudd AG, Wolfe CD. Prevalence of post stroke cognitive impairment: South London Stroke Register 1995-2010. Stroke (2013) 44:138-45. doi:10.1161/STROKEAHA.112.670844

5. Tatemichi TK, Desmond DW, Stern Y, Paik M, Sano M, Bagiella E. Cognitive impairment after stroke: frequency, patterns, and relationship to functional abilities. J Neurol Neurosurg Psychiatry (1994) 57:202-7. doi:10.1136/jnnp. 57.2.202

6. Nys GMS, van Zandvoort MJE, de Kort PLM, Jansen BPW, de Haan EHF, Kappelle LJ. Cognitive disorders in acute stroke: prevalence and clinical determinants. Cerebrovasc Dis (2007) 23:408-16. doi:10.1159/000101464

7. Jaillard A, Naegele B, Trabucco-Miguel S, LeBas JF, Hommel M. Hidden dysfunctioning in subacute stroke. Stroke (2009) 40:2473-9. doi:10.1161/ STROKEAHA.108.541144

8. Middleton LE, Lam B, Fahmi H, Black SE, Mcllroy WE, Stuss DT, et al. Frequency of domain-specific cognitive impairment in sub-acute and chronic stroke. NeuroRehabilitation (2014) 34:305-12. doi:10.3233/NRE131030

9. Dafer RM, Rao M, Shareef A, Sharma A. Post-stroke depression. Top Stroke Rehabil (2008) 15:13-21. doi:10.1310/tsr1501-13

10. Nys GMS, van Zandvoort MJE, de Kort PLM, van der Worp HB, Jansen BPW, Algra A, et al. The prognostic value of domain-specific cognitive abilities in acute first-ever stroke. Neurology (2005) 64:821-7. doi:10.1212/01.WNL. 0000152984.28420.5A

11. Paolucci S, Matano A, Bragoni M, Coiro P, De Angelis D, Fusco FR, et al. Rehabilitation of left brain-damaged ischemic patients: the role of comprehensionlanguage deficits. CerebrovascDis (2005) 20:400-6.doi:10.1159/000088671

12. Hochstenbach JB, Anderson PG, van Limbeek J, Mulder TT. Is there a relation between neuropsychologic variables and quality of life after stroke? Arch Phys Med Rehabil (2001) 82:1360-6. doi:10.1053/apmr.2001.25970

13. Hachinski V, Iadecola C, Petersen RC, Breteler MM, Nyenhuis DL, Black SE, et al. National Institute of Neurological Disorders and Stroke-Canadian Stroke Network vascular cognitive impairment harmonization standards. Stroke (2006) 37:2220-41. doi:10.1161/01.STR.0000237236.88823.47

14. Magni E, Binetti G, Bianchetti A, Rozzini R, Trabucchi M. Mini-Mental State Examination: a normative study in Italian elderly population. Eur J Neurol (1996) 3:198-202. doi:10.1111/j.1468-1331.1996.tb00423.x

15. Cumming TB, Bernhardt J, Linden T. The Montreal cognitive assessment short cognitive evaluation in a large stroke trial. Stroke (2011) 42:2642-4. doi:10.1161/STROKEAHA.111.619486

16. Demeyere N, Riddoch MJ, Slavkova ED, Jones K, Reckless I, Mathieson P, et al. Domain-specific versus generalized cognitive screening in acute stroke. J Neurol (2016) 263:306-15. doi:10.1007/s00415-015-7964-4

17. Tombaugh TN, McIntyre NJ. The mini-mental state examination: a comprehensive review. J Am Geriatr Soc (1992) 40:922-35. doi:10.1111/j.1532-5415. 1992.tb01992.x

18. Nys GMS, van Zandvoort MJE, de Kort PLM, Jansen BPW, Kappelle LJ, de Haan EHF. Restrictions of the mini-mental state examination in acute stroke. Arch Clin Neuropsychol (2005) 20:623-9. doi:10.1016/j.acn.2005. 04.001

19. Bour A, Rasquin S, Boreas A, Limburg M, Verhey F. How predictive is the MMSE for cognitive performance after stroke? J Neurol (2010) 257:630-7. doi:10.1007/s00415-009-5387-9

20. Dong Y, Sharma VK, Chan BPL, Venketasubramanian N, Teoh HL, Seet RCS, et al. The Montreal Cognitive Assessment (MoCA) is superior to the MiniMental State Examination (MMSE) for the detection of vascular cognitive impairment after acute stroke. J Neurol Sci (2010) 299:15-8. doi:10.116/j.jns.2010. 08.051

21. Arciniegas DB, Kellermeyer GF, Bonifer NM, Anderson-Salvi KM, Anderson CA. Screening for cognitive decline following single known stroke using the mini-mental state examination. Neuropsychiat Dis Treat (2011) 7:189-96. doi:10.2147/NDT.SI7886

22. Godefroy O, Fickl A, Roussel M, Auribault C, Bugnicourt JM, Lamy C, et al. Is the montreal cognitive assessment superior to the mini-mental state examination to detect post stroke cognitive impairment? Stroke (2011) 42:1712-6. doi:10.1161/STOKEAHA.110.606277

23. Barnay JL, Wauquiez G, Bonnin-Koang HY, Anquetil C, Pérennou D, Piscitelli C, et al. Feasibility of the cognitive assessment scale for stroke patients (CASP) vs. MMSE and MoCa in aphasic left hemispheric stroke patients. Ann Phys Rehabil Med (2014) 57:422-35. doi:10.1016/j.rehab.2014.05.010

24. Jacquin A, Binquet C, Rouaud O, Graule-Petot A, Daubail B, Osseby GV, et al. Post-stroke cognitive impairment: high prevalence and determining factors in a cohort of mild stroke. J Alzheimers Dis (2014) 40:1029-38. doi:10.3233/ JAD-131580

25. Benaim C, Barnay JL, Wuaquiez G, Bonnin-Koang HY, Anquetil C, Pérennou D, et al. The cognitive assessment scale for stroke patients (CASP) vs. MMSE and MoCa in non-aphasic hemispheric stroke patients. Ann Phys Rehabil Med (2015) 58:78-85. doi:10.1016/j.rehab.2014.12.001

26. Zhang H, Zhang XN, Zhang HL, Huang L, Chi QQ, Zhang X, et al. Differences in cognitive profiles between traumatic brain injury and stroke: a comparison of the Montreal Cognitive Assessment and Mini-Mental State Examination. Chin J Traumatol (2016) 19:271-4. doi:10.1016/j.cjtee.2015.03.007

27. Delavaran H, Jönsson AC, Lövkvist H, Iwarsson S, Elmståhl S, Norrving B, et al. Cognitive function in stroke survivors: a 10-year follow-up study. Acta Neurol Scand (2017) 136:187-94. doi:10.1111/ane.12709

28. Arba F, Quinn T, Hankey GJ, Ali M, Lees KR, Inzitari D. Cerebral small vessel disease, medial temporal lobe atrophy, and cognitive status in patients with ischaemic stroke and transient ischaemic attack. Eur J Neurol (2017) 24:276-82. doi:10.1111/ene.13191

29. Stephan BCM, Minett T, Muniz-Terrera G, Harrison SL, Matthews FE, Brayne C. Neuropsychological profiles of vascular disease and risk of dementia: implications for defining vascular cognitive impairment no dementia (VCI-ND). Age Ageing (2017) 46:755-60. doi:10.1093/ageing/afx016

30. You S, Wang X, Lindley RI, Robinson T, Anderson CS, Cao Y, et al. Early cognitive impairment after intracerebral hemorrhage in the INTERACT1 Study. Cerebrovasc Dis (2017) 44:320-4. doi:10.1159/000481443

31. Tan HH, Xu J, Teoh HL, Chan BPL, Seet RCS, Venketasubramanian N, et al. Decline in changing Montreal Cognitive Assessment (MoCA) scores is associated with post-stroke cognitive decline determined by a foral neuropsychological evaluation. PLoS One (2017) 12(3):e0173291. doi:10.1371/journal. pone. 0173291

32. Fu C, Jin X, Chen B, Niu H, Guo R, Chen Z, et al. Comparison of the mini-mental state examination and montreal cognitive assessment executive subtest in detecting post-stroke cognitive impairment. Geriatr Gerontol Int (2017) 17(12):2329-35. doi:10.1111/ggi.13069

33. Wang A, Liu J, Meng X, Li J, Wang H, Wang Y, et al. Association between oxidized low-density lipoprotein and cognitive impairment in patients with ischemic stroke. Eur J Neurol (2018) 25(1):185-91. doi:10.1111/ene.13497

34. National Institute for Clinical Excellence. Stroke Rehabilitation: Long-Term Rehabilitation after Stroke. NICE Clinical Guidelines CG162. London: National Institute for Health and Clinical Excellence (2013).

35. Demeyere N, Riddoch MJ, Slavkova ED, Bickerton WL, Humphreys GW. The Oxford Cognitive Screen (OCS): validation of a stroke-specific short cognitive screening tool. Psychol Assess (2015) 27:883-94. doi:10.1037/pas0000082

36. Bamford J, Sandercock P, Dennis M, Burn J, Warlow C. Classification and natural history of clinically identifiable subtypes of cerebral infarction. Lancet (1991) 337:1521-6. doi:10.1016/0140-6736(91)93206-O

37. Brott T, Adams HP, Olinger CP, Marler JR, Barsan WG, Biller J, et al. Measurements of acute cerebral infarction: a clinical examination scale. Stroke (1989) 20:864-70. doi:10.1161/01.STR.20.7.864

38. Mancuso M, Varalta V, Sardella L, Capitani D, Zoccolotti P, Antonucci G, et al. Italian normative data for a stroke specific cognitive screening tool: the Oxford Cognitive Screen (OCS). Neurol Sci (2016) 37:1713-21. doi:10.1007/ s10072-016-2650-6

39. Pendlebury ST, Cuthbertson FC, Welch SJV, Mehta Z, Rothwell PM. Underestimation of cognitive impairment by mini-mental state examination versus the montreal cognitive assessment in patients with transient ischemic attack and stroke. A population-based study. Stroke (2010) 41:1290-3. doi:10.1161/STROKEAHA.110.579888

40. Chan E, Khan S, Oliver R, Gill SK, Werring DJ, Cipolotti L. Underestimation of cognitive impairments by the Montreal Cognitive Assessment (MoCA) in an acute stroke unit population. J Neurol Sci (2014) 343:176-9. doi:10.1016/j.jns.2014. 05.005

41. Nasreddine ZS, Phillips NA, Bédirian V, Charbonneau S, Whitehead V, Collin I, et al. The Montreal Cognitive Assessment, MoCA: a brief screening tool for mild cognitive impairment. J Am Geriatr Soc (2005) 53:695-9. doi:10.1111/j.1532-5415.2005.53221.x 
42. Cumming TB, Churilov L, Linden T, Bernhardt J. Montreal cognitive assessment and mini-mental state examination are both valid cognitive tools in stroke. Acta Neurol Scand (2013) 128:122-9. doi:10.1111/ane.12084

43. Lees R, Selvarajah J, Fenton C, Pendlebury ST, Langhorne P, Stott DJ, et al. Test accuracy of cognitive screening tests for diagnosis of dementia and multidomain cognitive impairment in stroke. Stroke (2014) 45:3008-18. doi:10.1161/STROKEAHA.114.005842/-/DC1

44. Burton L, Tyson SF. Screening for cognitive impairment after stroke: a systematic review of psychometric properties and clinical utility. J Rehabil Med (2015) 47:193-203. doi:10.2340/16501977-1930

45. Bickerton WL, Demeyere N, Francis D, Kumar V, Remoundou M, Balani A, et al. The BCoS cognitive profile screen: utility and predictive value for stroke. Neuropsychology (2015) 29:638-48. doi:10.1037/ neu0000160

46. Von Campe G, Regli F, Bogousslavsky J. Heralding manifestations of basilar artery occlusion with lethal or severe stroke. J Neurol Neurosurg Psychiatry (2003) 74:1621-6. doi:10.1136/jnnp.74.12.1621
47. Kreiter KT, Copeland D, Bernardini GL, Bates JE, Peery S, Claassen J, et al Predictors of cognitive dysfunction after subarachnoid hemorrhage. Stroke (2002) 33:200-8. doi:10.1161/hs0102.101080

Conflict of Interest Statement: The authors declare that the research was conducted in the absence of any commercial or financial relationships that could be construed as a potential conflict of interest.

Copyright $@ 2018$ Mancuso, Demeyere, Abbruzzese, Damora, Varalta, Pirrotta, Antonucci, Matano, Caputo, Caruso, Pontiggia, Coccia, Ciancarelli, Zoccolotti and The Italian OCS Group. This is an open-access article distributed under the terms of the Creative Commons Attribution License (CC BY). The use, distribution or reproduction in other forums is permitted, provided the original author(s) and the copyright owner are credited and that the original publication in this journal is cited, in accordance with accepted academic practice. No use, distribution or reproduction is permitted which does not comply with these terms. 